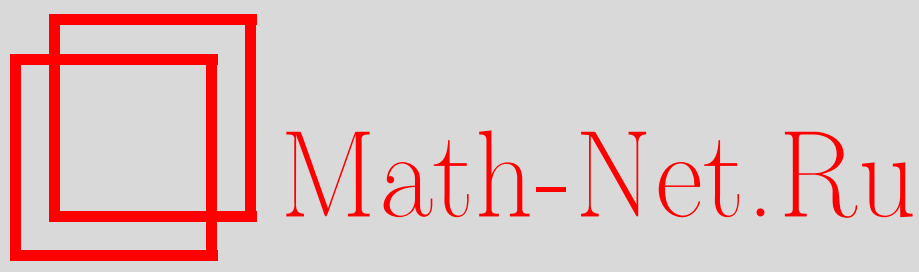

О. С. Якимова, Правильные изометрии слабо симметрических пространств, $M a$ тем. сб., 2002, том 193, номер 1, 143-156

DOI: https://doi.org/10.4213/sm624

Использование Общероссийского математического портала Math-Net.Ru подразумевает, что вы прочитали и согласны с пользовательским соглашением

http: //www. mathnet.ru/rus/agreement

Параметры загрузки:

IP : 52.87 .193 .239

26 апреля 2023 г., $12: 32: 54$ 
УДК 512.81

\author{
О.С. Якимова
}

\title{
Правильные изометрии слабо симметрических пространств
}

\begin{abstract}
В работе введено понятие правильного диффеоморфизма риманова многообразия. Такие диффеоморфизмы определяют на многообразии слабо симметрическую структуру. Для слабо симметрических римановых многообразий, являющихся однородными пространствами полупростых групп Ли, получена полная классификация правильных диффеоморфизмов (изометрий).

Библиографияя: 10 названий.
\end{abstract}

\section{§1. Введение}

Пусть $G$ - вешественная группа Ли, $K$ - ее компактная подгруппа, причем однородное пространство $M=G / K$ связно. Будем вначале предполагать, что действие $G: M$ эффективно, т.е. группа $K$ не содержит нетривиальных нормальных подгрупп группы $G$. Тогда группу $G$ можно рассматривать как подгруппу группы $\operatorname{Diff}(M)$ всех диффеоморфизмов многообразия $M$. Пусть $s-$ диффеоморфизм многообразия $M$.

ОПРЕДЕЛЕНИЕ 1. Однородное пространство $M$ называется слабо симметрическим относительно $s$, если выполнены следующие условия:

$$
\begin{gathered}
s G s^{-1}=G, \\
\forall x, y \in M \quad \exists g \in G: g x=s y, \quad g y=s x .
\end{gathered}
$$

Однородное пространство $M$ называется слабо симметрическим, если оно слабо симметрично относительно некоторого диффеоморфизма $s$.

Группу $\langle G, s\rangle \subset \operatorname{Diff}(M)$, порожденную группой $G$ и диффеоморфизмом $s$, обозначим через $\widehat{G}$.

Понятие слабо симметрического пространства впервые появилось в работе Сельберга [1]. Там также требовалось выполнение условия $s^{2} \in G$. Для большей обшности мы пока от него откажемся. Отметим, что доказательства всех основных результатов работ [2] и [3] не используют этого условия. Как будет доказано в дальнейшем, в случае полупростой группы $G$ данное определение эквивалентно определению Сельберга.

На слабо симметрическом пространстве $M$ можно ввести $G$-инвариантную риманову метрику, которая автоматически будет $\widehat{G}$-инвариантной; поэтому к нему можно применять результаты работы [3].

Работа выполнена при поддержке CRDF (грант № RM1-2088).

$$
\text { (C) О.С. Якимова } 2002
$$


ОПРЕДЕЛЕНИЕ 2. Диффеоморфизм $s$ многообразия $M$ называется $п р а в и л ь н ы м$, если он удовлетворяет условиям (1) и (2).

Цель настоящей работы - описать все правильные диффеоморфизмы (изометрии) слабо симметрических однородных пространств полупростых групп Ли.

Если правильный диффеоморфизм умножить слева или справа на элемент группы $G$, то он останется правильным. Поэтому задача сводится к описанию смежных классов в $\operatorname{Diff}(M) / G$, состоящих из правильных диффеоморфизмов. Такие классы будем называть правильными. В каждом классе есть элементы, оставляющие на месте точку $e K$. Если $s(e K)=e K$, то $s(g K)=\sigma(g) K$, где $\sigma \in$ Aut $G$, $\sigma(g)=s g s^{-1}$. Для автоморфизма $\sigma$ выполнено следуюшее условие:

(i) $\sigma(K)=K$.

Множество автоморфизмов (внутренних автоморфизмов) группы $G$, удовлетворяющих условию (i), обозначим через $\operatorname{Aut}(G, K)(\operatorname{Int}(G, K))$.

Любому автоморфизму $\sigma$ группы $G$, удовлетворяющему условию (i), соответствует диффеоморфизм $s$ многообразия $M$, определяемый по формуле

$$
s(g K)=\sigma(g) K .
$$

Обозначим сопряжение элементом $g(g \in G)$ в группе $G$ через $a(g)$. Автоморфизмам $\sigma$ и $\tau$ групшы $G$, удовлетворяющим условию (i), соответствуют лежащие в одном смежном классе диффеоморфизмы многообразия $M$ тогда и только тогда, когда $\sigma=a(k) \tau, k \in K$.

Пусть $\mathfrak{g}$ и $\mathfrak{k}$ - касательные алгебры групп $G$ и $K$ соответственно. Касательное пространство $T_{e K} M$ канонически отождествляется с $\mathfrak{g} / \mathfrak{k}$. Группа $\operatorname{Aut}(G, K)$ естественным образом действует на $\mathfrak{g} / \mathfrak{k}$. Если $\sigma \in \operatorname{Aut}(G, K)$ и $\eta \in \mathfrak{g}$, то $\sigma(\eta+\mathfrak{k}):=$ $d \sigma(\eta)+\mathfrak{k}$.

В работе [3] доказывается, что для элементов группы $\operatorname{Aut}(G, K)$ условие (2) равносильно следуюшему:

(ii) $\forall \xi \in \mathfrak{g} / \mathfrak{k} \quad \exists k \in K:(\operatorname{Ad} k) \xi=-\sigma(\xi)$.

ОПРЕДЕЛЕНИЕ 3. Автоморфизм $\sigma$ группы $G$ называется правильным автоморфизмом пары $(G, K)$, если он удовлетворяет условиям (i), (ii).

Иногда в тех случаях, когда понятно, о какой компактной подгруппе идет речь, мы будем называть правильные автоморфизмы пары $(G, K)$ правильньпи автоморфизмами группы $G$. Описание правильных классов в $\operatorname{Diff}(M) / G$ равносильно описанию правильных классов в $\operatorname{Aut}(G, K) / a(K)$.

Орбиты групшы $K$ в векторном пространстве $\mathfrak{g} / \mathfrak{k}$ разделяются полиномиальными инвариантами (см. [4]). Поэтому условие (ii) равносильно следующему:

(ii $\left.{ }^{\prime}\right)$ если $f \in \mathbb{R}[\mathfrak{g} / \mathfrak{k}]^{K}$ - однородный $K$-инвариантньй многочлен, то $\sigma(f)=$ $(-1)^{\operatorname{deg} f} f$.

ПРЕДЛОЖЕНИЕ 1 [5; лемма 1]. Пусть $K^{0}$ - связная компонента группь $K$, $G^{0}$ - связная компонента группьц $G, \sigma$ - правильный автоморфизм пары $(G, K)$. Тогда найдется такой әлемент $k \in K$, что $a(k) \sigma$ - правильньй автоморфизм пары $\left(G^{0}, K^{0}\right)$.

Начиная с этого момента, мы будем рассматривать только однородные пространства связных полупростых груп, причем будем предполагать, что подгруппа $K$ связна.

Предположим теперь, что действие $G:(G / K)$ не обязательно эффективно, но локально эффективно, т.е. ядро неэффективности $N$ дискретно. Тогда $N=$ 
$Z(G) \cap K$, где $Z(G)$ - центр группы $G$. Определение 2 переносится без изменений на этот более общий случай. Заметим, что если $\sigma \in \operatorname{Aut}(G, K)$, то

$$
\sigma(N)=\sigma(Z(G) \cap K)=\sigma(Z(G)) \cap \sigma(K)=Z(G) \cap K=N .
$$

Так как группа $N$ действует на векторном пространстве $\mathfrak{g} / \mathfrak{k}$ тривиально, то любому правильному автоморфизму пары $(G, K)$ соответствует правильньй автоморфизм пары $(G / N, K / N)$ и, следовательно, правильный диффеоморфизм многообразия $M=G / K=(G / N) /(K / N)$. Пусть $\widetilde{G}$ - односвязная накрьвающая группы $G$. Группы Aut $G$ и $\operatorname{Aut}(G / N)$ можно рассматривать как подгруппы группы Aut $\widetilde{G}$. Если в этой групше Aut $G=\operatorname{Aut}(G / N)$, то множества правильных автоморфизмов пар $(G, K)$ и $(G / N, K / N)$ совпадают.

Пусть $\widetilde{K}$ - связная подгруппа группы $\widetilde{G}$ с касательной алгеброй $\mathfrak{k}$. Тогда любой правильный автоморфизм пары $(G, K)$ поднимается до правильного автоморфизма пары $(\widetilde{G}, \widetilde{K})$, а если Aut $G=\operatorname{Aut} \widetilde{G}(=\operatorname{Aut} \mathfrak{g})$, то множества правильных автоморфизмов этих пар совпадают. Действительно, группа $G$ является фактором групшы $\widetilde{G}$ по некоторой дискретной центральной подгруппе $Z$ и $K=\widetilde{K} /(\widetilde{K} \cap Z)$. Группы $K$ и $\widetilde{K}$ действуют на $\mathfrak{g} / \mathfrak{k}$ так же, как группа $\widetilde{K} /(\widetilde{K} \cap Z(\widetilde{G}))$. С другой стороны, если $\sigma$ является автоморфизмом групшы $G$, то $\sigma(K)=K$ тогда и только тогда, когда $\sigma(\mathfrak{k})=\mathfrak{k}$.

Из всех групп $G$ с данной касательной алгеброй мы будем рассматривать только ту, которая является вешественной формой односвязной комплексной группы Ли. Для такой групшы $G$ группы Aut $G$ и Aut $\mathfrak{g}$ совпадают.

Сушествует теория, позволяюшая перейти к комплексным слабо симметрическим пространствам и их правильньм автоморфизмам (см. определение 4). Задача будет сначала решена для комплексных пар. Множество правильных автоморфизмов пары $\left(G_{\mathbb{C}}, K_{\mathbb{C}}\right)$ является объединением некоторого количества связных компонент группы $\operatorname{Aut}\left(G_{\mathbb{C}}, K_{\mathbb{C}}\right)$. А правильными автоморфизмами пары $(G, K)$ будут те и только те правильные автоморфизмы пары $\left(G_{\mathbb{C}}, K_{\mathbb{C}}\right)$, которые сохраняют группу $G$.

\section{§2. Действие группы $N(K)$ на многообразии $M$}

Группа $N(K)$ (нормализатор подгруппы $K$ в групше $G$ ) действует на пространстве $G / K$ умножениями справа. Ядро неэффективности этого действия есть группа $K$. Это действие перестановочно с действием группы $G$. Обозначим диффеоморфизм многообразия $M$, соответствуюший элементу $n \in N(K)$, через $\Upsilon_{n}$, т.е.

$$
\Upsilon_{n}(g K)=g K n^{-1}=g n^{-1} K .
$$

Лемма 1. Пусть $s-$ правильный диффеоморфизм многообразия $M$, $n \in N(K)$. Тогда $s \Upsilon_{n} s^{-1}=\Upsilon_{n}^{-1}$ и диффеоморфизм $s \Upsilon_{n}$ тоже правильный.

ДокАЗАТЕльСтво. Рассмотрим точки $x$ и $y=\Upsilon_{n} x$ многообразия $M$. Найдется такой элемент $g \in G$, что

$$
g x=s y, \quad g y=s x .
$$

Тогда $s x=g \Upsilon_{n} x=\Upsilon_{n}(g x)=\Upsilon_{n} s \Upsilon_{n} x$. Поскольку это верно для любой точки $x \in M$, то $\Upsilon_{n} s \Upsilon_{n}=s$. 
Пусть $x, y$ - произвольные точки многообразия $M$. По определению 1 найдется такой элемент $g \in G$, что

$$
g x=s\left(\Upsilon_{n} y\right), \quad g\left(\Upsilon_{n} y\right)=s x
$$

Тогда

$$
s \Upsilon_{n} x=\Upsilon_{n}^{-1} s x=\Upsilon_{n}^{-1}\left(g \Upsilon_{n} y\right)=g y, \quad s \Upsilon_{n} y=g x
$$

СЛЕДСТВИЕ 1. Пусть $\sigma$ - правильный автоморфизм пары $(G, H)$ и $n \in$ $N(K)$. Тогда $\sigma(n) \equiv n^{-1} \bmod K$ и автоморфизм $a(n) \sigma$ тожсе правильный.

ДоказАТЕЛЬСтво. Рассмотрим соответствующий диффеоморфизм $s$ многообразия $M$. Имеем

$$
e K=s(e K)=\Upsilon_{n} s \Upsilon_{n}(e K)=\Upsilon_{n}\left(\sigma\left(n^{-1}\right) K\right)=\sigma\left(n^{-1}\right) n^{-1} K
$$

откуда $\sigma(n) n \in K$. Диффеоморфизм $n s \Upsilon_{n}^{-1}=\Upsilon_{n} n s$ является правильным и оставляет точку $e K$ на месте. Ему соответствует автоморфизм группы $G$, равньй $a(n) \sigma$.

Правильные автоморфизмы $\sigma$ и $a(n) \sigma$ будем называть әквивалентными.

СлеДСТВИЕ 2. Групnа $N(K) / K$ абелева, и орбить гpynn $K$ u $N(K)$ в $\mathfrak{g} / \mathfrak{k}$ совпадают.

ДоКАЗАТЕЛЬСТво. Из леммы 1 следует, что инверсия является автоморфизмом группы $N(K) / K$. Следовательно, эта группа абелева.

Пусть $n \in N(K)$ и $\sigma-$ правильный автоморфизм группы $G$. Тогда для $\xi \in \mathfrak{g} / \mathfrak{k}$ имеем

$$
\begin{aligned}
(\operatorname{Ad} n) \xi & =(\operatorname{Ad} n) \sigma\left(\sigma^{-1}(\xi)\right)=-\left(\operatorname{Ad} k_{1}\right) \sigma^{-1}(\xi)=-\left(\operatorname{Ad} k_{1}\right)\left(-\left(\operatorname{Ad} k_{2}\right) \xi\right) \\
& =\left(\operatorname{Ad}\left(k_{1} k_{2}\right)\right) \xi \quad\left(k_{1}, k_{2} \in K\right)
\end{aligned}
$$

СлеДСТВИЕ 3. Группа $N(K)$ действует тривиально на алгебре $\mathbb{R}[\mathfrak{g} / \mathfrak{k}]^{K}$.

\section{§3. Комплексные слабо симметрические пространства}

Сформулируем несколько утверждений работы [2] и их следствий, позволяющих перейти к однородньм пространствам комплексных групп Ли.

Пусть $G$ - полупростая комплексная алгебраическая группа, $X$ - неприводимое аффинное алгебраическое многообразие. Пусть группа $G$ действует транзитивно и эффективно на $X$, и пусть $s$ - автоморфизм алгебраического многообразия $X$, нормализующий группу $G$. Определим действие групшы $\widehat{G}=\langle G, s\rangle$ на многообразии $X \times X$ следуюшим образом:

$$
\begin{aligned}
& s(x, y)=(s y, s x) \\
& g(x, y)=(g x, g y)
\end{aligned}
$$

ОПРЕДЕЛЕНИЕ 4. Однородное пространство $X$ называется слабо симметрическим относительно $s$, если автоморфизм $s$ действует тривиально на алгебре $\mathbb{C}[X \times X]^{G}$. Однородное пространство $X$ называется слабо симметрическим, если оно слабо симметрично относительно некоторого автоморфизма $s$ алгебраического многообразия $X$. Этот автоморфизм $s$ будем называть правильнымм автоморфизмом алгебраического многообразия $X$. 
ОПРЕДЕЛЕНИЕ 5. Однородное пространство $G / H$ (подгруппа $H$, пара $(G, H)$ ) называется сферическим (сферической), если в $G / H$ есть открытая орбита борелевской подгруппы группы $G$.

Напомним, что комплексная (или вещественная) линейная группа Ли, имеющая конечное число связных компонент, называется редуктивной, если она вполне приводима. В частности, любая полупростая группа редуктивна.

Пусть $G$ и $H \subset G$ - связные редуктивные алгебраические группы, $K$ - компактная вещественная форма группы $H, G_{0}$ - связная компонента некоторой вещественной формы группы $G$, содержащая $K, M=G_{0} / K, X=G / H$.

Лемма 2. Пусть $N$ - ядро неәффективности действия $G: X, a N_{0}-$ ядро неәффективности действия $G_{0}: M$. Тогда, если одна из групп $N, N_{0}$ дискретна, то $N=N_{0}$. B частности, действие $G: X$ эффективно тогда и только тогда, когда эффективно действие $G_{0}: M$.

ДокАЗАТЕльство. Если одна из групп $N, N_{0}$ дискретна, то они обе дискретны и, следовательно, являются подгруппами центров групп $G$ и $G_{0}$. Более точно,

$$
N=Z(G) \cap H=Z(G) \cap Z(H), \quad N_{0}=Z\left(G_{0}\right) \cap K=Z\left(G_{0}\right) \cap Z(K) .
$$

Как известно, $Z(K)=Z(H)$, а $Z\left(G_{0}\right)=Z(G) \cap G_{0}$. Поэтому

$$
N_{0}=Z(G) \cap Z(K)=Z(G) \cap Z(H)=N .
$$

Инволютивный автоморфизм связной редуктивной комплексной алгебраической группы $G$ называется инволюиией Вейля, если он действует как инверсия на некотором максимальном торе этой группы. Известно, что инволюции Вейля существуют и все они сопряжены при помощи внутренних автоморфизмов.

Теорема 1 [2; теоремы 2.2, 3.3, 4.2]. Пусть действие $G: X$ әффективно. Тогда

1) если $s$ - такой автоморфизм алгебраического многообразия $X$, что $s H s^{-1}=H$ u $s M=M$, то слабая симметричность однородного пространства $M$ относительно s равносильна слабой симметричности однородного пространства $X$ относительно s;

2) однородное пространство $X$ слабо симметрично тогда и только тогда, когда оно сферично. Более точно, в этом случае оно слабо симметрично относительно следующего автоморфизма $t$ :

$$
t(g H)=\theta(g) H
$$

где $\theta$ - инволючия Вейля группь $G$, ограничение которой на подгруппу $H$ является инволюиией Вейля группь $Н$. При әтом инволюиию $\theta$ можно вьбрать так, чтобь она сохраняла группу $G_{0}$. (В дальнейшем эту инволюцию Вейля группь $G$ всегда будем обозначать символом $\theta$.)

Рассматриваемые нами автоморфизмы алгебраического многообразия $X$ определяют автоморфизмы группы $G$, а именно, автоморфизму $s \in$ Aut $X$ соответствует автоморфизм $g \mapsto \sigma(g)=s g s^{-1}$. И наоборот, каждому элементу группы $\operatorname{Aut}(G, H)$ можно сопоставить автоморфизм алгебраического многообразия $G / H$. Определим множество правильных автоморфизмов пары $(G, H)$ аналогично вещественному случаю. Как и в вещественном случае, мы будем предполагать, что ядро неэффективности действия $G: X$ дискретно. 
Условия (ii) и (ii') в комплексном случае вьглядят так:

(ii) $\mathbb{C}$ если $\xi \in \mathfrak{g} / \mathfrak{h}$ и $H \xi=\overline{H \xi}$, то существует такой элемент $h \in H$, что $(\operatorname{Ad} h) \xi=$ $-\sigma(\xi)$

$\left(\mathrm{ii}^{\prime}\right)_{\mathbb{C}}$ если $f \in \mathbb{C}[\mathfrak{g} / \mathfrak{h}]^{H}$ - однородный $H$-инвариантный многочлен, то $\sigma(f)=$ $(-1)^{\operatorname{deg} f} f$.

Пусть $R$ - неприводимое представление группы $G, \sigma \in \operatorname{Aut} G$. Обозначим через $R^{\sigma}=R \circ \sigma$ подкрученное на $\sigma$ представление $R$ и через $R^{*}$ - представление, двойственное к $R$. Пусть группа $G$ действует на аффинном алгебраическом многообразии $X$. Тогда как $G$-модуль алгебра $\mathbb{C}[X]$ имеет следующий вид:

$$
\mathbb{C}[X]=\bigoplus_{R}(V(R) \otimes U(R)),
$$

где $R$ пробегает все неприводимые представления $G, G$ действует на $V(R)$ при помощи $R$, а на $U(R)$ тривиально.

Хорошо известно, что если $X$ - сферическое однородное пространство группы $G$, то $\operatorname{dim} U(R) \leqslant 1$. Множество тех неприводимых представлений, для которых $U(R)=1$, обозначим через $\mathfrak{R}$.

ПРЕДЛОЖЕНИЕ 2. Пусть $X=G / H$ - сферическое однородное пространство группь $G, \mathbb{C}[X]=\bigoplus_{R \in \mathfrak{R}} V(R)$ - разлоэсение $\mathbb{C}[X]$ в прямую сумму неприводимых представлений группь $G$ и $\sigma$ - правильный автоморфизм парь $(G, H)$. Тогда выполнено условие

(iii) $R^{\sigma}=R^{*}$ для любого $R \in \mathfrak{R}$.

ДоказАТЕЛЬство. Пусть $s$ - автоморфизм алгебраического многообразия $X$, соответствующий $\sigma$. По определению правильного автоморфизма пары многообразие $X$ слабо симметрично относительно $s$. Рассмотрим действие $s$ на алгебре

$$
\mathbb{C}[X \times X]=\mathbb{C}[X] \otimes \mathbb{C}[X]=\bigoplus_{R, S \in \mathfrak{R}}(V(R) \otimes V(S)) .
$$

Как известно, в каждом слагаемом вида $V(R) \otimes V\left(R^{*}\right)$ существует ненулевой $G$-инвариант. Поэтому имеем:

$$
\forall R \in \Re \quad V(R) \otimes V\left(R^{*}\right)=s\left(V(R) \otimes V\left(R^{*}\right)\right)=V\left(\left(R^{*}\right)^{\sigma}\right) \otimes V\left(R^{\sigma}\right) .
$$

В частности, $V\left(R^{\sigma}\right)=V\left(R^{*}\right)$.

Пусть слабо симметрическое пространство $M=G_{0} / K$ является вешественной формой сферического пространства $X=G / H$ (в смысле условия теоремы 1 ), причем группа $G$ односвязна. Пусть $\sigma$ - правильный автоморфизм групшы $G_{0}$. Он продолжается до автоморфизма групшы $G$, сохраняюшего подгруппу $H$, и, следовательно, определяет автоморфизм однородного пространства $X$. Таким образом (по теореме 1), существует биекция между множеством правильных автоморфизмов группы $G_{0}$ и множеством правильных автоморфизмов группы $G$, удовлетворяющих условию

(iv) $\sigma\left(G_{0}\right)=G_{0}$.

Если условие (iv) выполнено, то $\sigma(K)=K$ тогда и только тогда, когда $\sigma(H)=H$.

Действие $N_{G_{0}}(K): \mathfrak{g}_{0} / \mathfrak{k}$ является вешественной формой действия $N(H): \mathfrak{g} / \mathfrak{h}$. Поэтому, как и в вешественном случае, инварианты групп $N(H)$ и $H$ в пространстве $\mathbb{C}[\mathfrak{g} / \mathfrak{h}]$ совпадают. В частности, если правильный автоморфизм пары $(G, H)$ умножить на $a(n)$, где $n \in N(H)$, то он останется правильным. Правильные автоморфизмы $\sigma$ и $a(n) \sigma(n \in N(H))$ будем назьвать әквивалентными. 


\section{§4. Правильные автоморфизмы комплексных сферических пар}

Пусть $(G, H)$ - сферическая пара ( $G$ и $H$ - связные комплексные алгебраические группы), причем группа $G$ односвязна и полупроста. В данном параграфе описаны правильные автоморфизмы пары $(G, H)$. В каждой связной компоненте группы Aut $G=$ Aut $\mathfrak{g}$ будет указан один правильный автоморфизм, если, конечно, он там есть. Тем самым будут перечислены все правильные автоморфизмы с точностью до эквивалентности.

Перед тем как перейти непосредственно к классификации, докажем утверждение, дающее полное описание всех правильных автоморфизмов.

Теорема 2. Пусть $(G, H)$ - локально әффективная сферическая пара, группа $G$ полупроста, $H$ редуктивна. Пусть $\sigma \in \operatorname{Aut}(G, H)$ удовлетворяет условию (iіi). Тогда $\sigma$ - правильный автоморфизм парьь $(G, H)$.

ДокАЗАТЕльство. Достаточно доказать, что $\sigma$ эквивалентен некоторому правильному автоморфизму. Множество $\sigma \operatorname{Int}(G, H)$ является объединением некоторого количества связных компонент алгебраической группы $\operatorname{Aut}(G, H)$. Следовательно, в нем есть элемент конечного порядка. Можно считать, что сам $\sigma$ имеет конечньй порядок. Будем также считать, что действие $G:(G / H)$ эффективно, т.е. $G \subset$ Aut $X$.

Автоморфизм $\sigma$ индуцирует автоморфизм многообразия $X: s(g H)=\sigma(g) H$. Легко видеть, что автоморфизм $s$ тоже имеет конечный порядок. Следовательно, группа $\widehat{G}=\langle G, s\rangle \subset$ Aut $X$ (подгруппа, порожденная группой $G$ и автоморфизмом $s$ ) редуктивна. Многообразие $X$ является сферическим однородньм пространством группы $\widehat{G}$. Более точно, $X=\widehat{G} / \widehat{H}$, где $\widehat{H}=\langle H, s\rangle$.

Пусть $\mathbb{C}[X]=\bigoplus_{R \in \mathfrak{R}} V(R)$ - разложение $\mathbb{C}[X]$ в прямую сумму неприводимых представлений групшы $G$. Из условия (iii) следует, что $s(V(R))=V\left(R^{*}\right)$. Теперь разложим $\mathbb{C}[X]$ в сумму неприводимых представлений группы $\widehat{G}$ :

$$
\mathbb{C}[X]=\bigoplus_{R \in \mathfrak{R}, R=R^{*}} V(R) \oplus \bigoplus_{R \in \mathfrak{R}, R \neq R^{*}}\left(V(R) \oplus V\left(R^{*}\right)\right) .
$$

Обозначим слагаемые $V(R) \oplus V\left(R^{*}\right)$ из второй суммы через $W(R)$. (На самом деле, во второй части суммирование производится по неупорядоченным парам $\left\{R, R^{*}\right\}$, но чтобы не вводить слишком громоздких обозначений, мы допускаем некоторую формальную неточность.)

Представление группы $\widehat{G}$ в каждом из пространств $V(R)$ и $W(R)$ является самосопряженным представлением групшы $\widehat{G}$. Если бы, например, представление $\widehat{G}: W(R)$ не было самосопряженным, то представление $\widehat{G}: W(R)^{*}$ тоже входило бы в $\mathbb{C}[X]$. Но представления группы $G$ в $W(R)$ и $W(R)^{*}$ изоморфны. Это противоречит сферичности пространства $G / H$.

Покажем, что эти представления ортогональны. Если $\widehat{G}: U-$ симплектическое неприводимое представление группы $\widehat{G}$, то на подпространстве $U^{\widehat{H}}$ тоже существует невырожденная кососимметричная форма (ограничение аналогичной формы со всего пространства $U$ ). Это означает, что $\operatorname{dim} U^{\widehat{H}}-$ четное число и, следовательно, такое представление не может входить в $\mathbb{C}[X]$.

Рассмотрим действие групшы $\widehat{G}: X \times X$, определенное формулами (3) и (4). Нужно доказать, что $\mathbb{C}[X \times X]^{G}=\mathbb{C}[X \times X]^{\widehat{G}}$. Напомним, что

$$
\mathbb{C}[X \times X]^{G}=\bigoplus_{R \in \Re, R=R^{*}} S^{2} V(R)^{G} \oplus \bigoplus_{R \in \mathfrak{R}, R \neq R^{*}}\left(S^{2} W(R)^{G} \oplus \Lambda^{2} W(R)^{G}\right)
$$


причем $\operatorname{dim} S^{2} V(R)^{G}=\operatorname{dim} S^{2} W(R)^{G}=\operatorname{dim} \Lambda^{2} W(R)^{G}=1$.

Как мы уже знаем, в пространствах $S^{2} V(R)$ и $S^{2} W(R)$ сушествуют ненулевые $\widehat{G}$-инварианты, т.е. $\operatorname{dim} S^{2} V(R)^{\widehat{G}}=\operatorname{dim} S^{2} W(R)^{\widehat{G}}=1$. А поскольку и $\operatorname{dim} S^{2} V(R)^{G}=\operatorname{dim} S^{2} W(R)^{G}=1$, эти пространства совпадают: $S^{2} V(R)^{\widehat{G}}=$ $S^{2} V(R)^{G}, S^{2} W(R)^{\widehat{G}}=S^{2} W(R)^{G}$. Так как перестановка аргументов $(x, y) \rightarrow$ $(y, x)$ действует на пространствах $S^{2} V(R)$ и $S^{2} W(R)$ тривиально, доказано, что автоморфизм $s$ действует тривиально на алгебре $\bigoplus S^{2} V(R)^{G} \oplus \bigoplus S^{2} W(R)^{G}$.

Посмотрим, как $s$ действует на оставшихся $G$-инвариантах. Пусть $\omega \in$ $\Lambda^{2} W(R)^{G}$.

Автоморфизм $s^{2}$ сохраняет каждое из представлений $V(R)$. Рассмотрим групшу $\left\langle G, s^{2}\right\rangle \subset \widehat{G}$. Как и группа $\widehat{G}$ она редуктивна и, поскольку имеет два линейно независимых инвариантных вектора в пространстве $W(R) \otimes W(R)$, сохраняет форму $\omega$. Следовательно, $s \omega= \pm \omega$. Предположим, что $s \omega=-\omega$. Тогда форма $\omega$ инвариантна относительно диагонального действия элемента $s$ на многообразии $X \times X$ $(s(x, y)=(s x, s y))$. Это означало бы, что $\operatorname{dim}(W(R) \otimes W(R))^{G}=2$ для диагонального действия группы $\widehat{G}$ на $X \times X$. Но это не так. На самом деле, как мы видели, данная размерность равна 1.

СлЕДСТВИЕ 1. Множество всех правильных автоморфизмов пары $(G, H)$ совпадает с обтединением тех связных компонент группь $\operatorname{Aut}(G, H)$, для которых выполнено условие (iii).

СлЕДСТВИЕ 2. Все замкнутые $H$-орбиты в $\mathfrak{g} / \mathfrak{h}$ симметричны относительно нуля тогда и только тогда, когда все неприводимые представления, входящие в разложсение $\mathbb{C}[G / H]$, самосопряжены.

ДокАЗАТЕльство. Оба утверждения равносильны тому, что тождественное преобразование является правильным автоморфизмом пары $(G, H)$.

В каждом конкретном случае для нахождения всех правильных автоморфизмов было бы достаточно найти группу $\operatorname{Aut}(G, H)$ и ранговую полугруппу (множество весов в $\left.\mathbb{C}[X]^{(B)}\right)$ или даже скорее ранговую группу $\Gamma(X)$ (множество весов в $\left.\mathbb{C}(X)^{(B)}\right)$. (Здесь $B$ - борелевская подгруппа группы $G$.)

Для решения последней задачи можно воспользоваться, например, методом Д. И. Панюшева, изложенным в статье [6]. Пусть $T \subset B$ - максимальный тор, $\theta-$ инволюция Вейля групшы $G$, действуюшая на нем инверсией. Подгруппу $H$ можно так заменить на сопряженную, чтобы были выполнены все следующие условия:

а) группы $B \cap H$ и $B \cap \theta(H)$ одновременно имеют минимально возможные размерности;

b) они обе нормализуются тором $T$;

c) $B \cap H-$ борелевская подгрупша редуктивной группы $H \cap \theta(H)$.

Если $H$ удовлетворяет условиям а) - c), то $H_{*}=H \cap \theta(H)$ - стабилизатор общего положения действия $H: \mathfrak{g} / \mathfrak{h}$, а $\Gamma(G / H)$ совпадает с аннулятором группы $H_{*} \cap T$.

Заметим, что в случае простой группы $G$ ранговые полугруппы уже известны и приведены в работе [7].

Но, оказывается, во всех остальных случаях существуют более простые способы нахождения правильных автоморфизмов.

Теперь перейдем к разбору конкретных пар.

ОПРЕДЕЛЕНИЕ 6. Сферическая пара $(G, H)$ называется неразложиммой, если она не представима в виде $\left(G=G^{1} \times G^{2}, H=H^{1} \times H^{2}\right)$, где пары $\left(G^{1}, H^{1}\right)$ и $\left(G^{2}, H^{2}\right)$ сфферические. 
Неразложимые сферические пары классифицированы в работах [7]-[10].

Лемма 3. Пусть $G=G^{1} \times \cdots \times G^{n}, H=H^{1} \times \cdots \times H^{n}$, әде пара $\left(G^{i}, H^{i}\right)$ неразложима для любого $i$, и $\sigma$ - правильный автоморфизм пары $(G, H)$. Тогда $\sigma\left(G^{i}\right)=G^{i}$.

ДоКАЗАТЕЛЬСтво. Если $\sigma$ переводит простой множитель $G_{1}^{i}$ групшы $G^{i}$ в простой множитель $G_{1}^{j}$ группы $G^{j}(i \neq j)$, то $\sigma$ переводит группу $H^{i}$ в группу $H^{j}$ (иначе $\sigma(H) \neq H$ ) и $G^{i}$ в $G^{j}$. В разложении $\mathbb{C}[X]$ на неприводимые представления групшы $G$ найдется представление, на котором группы $G^{k}$ при $k \neq i$ действуют тривиально. Обозначим его через $R$. Тогда на пространстве $V\left(R^{*}\right)$ группа $G^{j}$ действует тривиально, а на $V\left(R^{\sigma}\right)$ нет.

Лемма 4. Пусть $G^{i}$ - простая нормальная подгруппа группь $G, H^{i}-$ проекиия на нее группы $H, \sigma$ - правильный автоморфизм. Если $H^{i} \neq G^{i}$, то $\sigma\left(G^{i}\right)=G^{i}$.

ДокАЗАТЕЛЬСтво. Сушествует неприводимое представление $R$ группы $G^{i}$, для которого $V(R)^{H^{i}} \neq 0$. Остальная часть доказательства аналогична доказательству предыдушей леммы.

Также отметим, что в условиях этих лемм ограничение правильного автоморфизма группы $G$ на группу $G^{i}$ будет правильным автоморфизмом для сферической пары $\left(G^{i}, H^{i}\right)$.

Из классификации, в частности, следует, что для неразложимой сферической пары $(G, H)$ группа $H^{i}$ может совпадать с группой $G^{i}$, только если $(G, H)$ является одной из пар:

1) симметрическая пара $(H \times H, H)$;

2) $\left(\mathrm{Sp}_{2} \times \mathrm{Sp}_{4} \times \mathrm{Sp}_{2}, \mathrm{Sp}_{2} \times \mathrm{Sp}_{2}\right)$;

3) $\left(\mathrm{Sp}_{2 n} \times \mathrm{Sp}_{2} \times \mathrm{Sp}_{2}, \mathrm{Sp}_{2 n-2} \times \mathrm{Sp}_{2}\right)$.

Но нетрудно убедиться в том, что в последних двух случаях существуют неприводимые представления групшы $G$, содержащие ненулевые $H$-инвариантные векторы, для которых условие (iii) не выполнено, если автоморфизм $\sigma$ не сохраняет простые компоненты. Укажем старшие веса этих представлений. В случае 2) это $\pi_{1}(1) \cdot \pi_{1}(2)$; а в случае 3 ) это $\pi_{1}(1) \cdot \pi_{1}(2)$ и $\pi_{1}(1) \cdot \pi_{1}(3)$. (Цифра в скобках соответствует номеру простой компоненты, а $\pi_{i}$ - фундаментальные веса соответствуюших групп. Подробнее по поводу этих обозначений см. [7], [4].)

ПРЕДЛОЖЕНИЕ 3. Пусть $\left(G=G^{1} \times G^{2}, H=H^{1} \times H^{2}\right)$ - разложсимая сферическая пара, $\sigma^{1}$ и $\sigma^{2}$ - автоморфизмы групп $G^{1}$ и $G^{2}$ соответственно. Автоморфизм $\sigma=\sigma^{1} \times \sigma^{2}$ правильный тогда и только тогда, когда $\sigma^{1}$ и $\sigma^{2}$ правильнье.

ДокАЗАТЕЛЬСТво. Имеем $\mathfrak{g} / \mathfrak{h}=\mathfrak{g}^{1} / \mathfrak{h}^{1} \oplus \mathfrak{g}^{2} / \mathfrak{h}^{2}$, причем группа $G^{i}$ действует на алгебре $\mathfrak{g}^{j}$ при $i \neq j$ тривиально. Поэтому условие (ii) $\mathbb{C}$ выполнено для автоморфизма $\sigma$ тогда и только тогда, когда оно выполнено для автоморфизмов $\sigma^{1}$ и $\sigma^{2}$.

Теперь укажем правильные автоморфизмы неразложимых сферических пар.

Пусть $\mathfrak{g}, \mathfrak{h}$ - касательные алгебры групп $G$ и $H$ соответственно. На алгебре $\mathfrak{g}$ существует $G$-инвариантное скалярное произведение. Пространство $\mathfrak{g} / \mathfrak{h}$ будем отождествлять с ортогональньм дополнением $\mathfrak{h}^{\perp}$ к подалгебре $\mathfrak{h}$ в алгебре $\mathfrak{g}$.

Отметим, что для симметрической пары инволюция, определяющая симметрическую подгруппу, всегда будет правильным автоморфизмом. Обозначим ее символом $\varphi$, инволюцию Вейля группы $G$ (из теоремы 1 ) - символом $\theta$, тождественный автоморфизм - символом id. 
Для пар с простой группой $G$ в статье $[7]$ приведено разложение $\mathbb{C}[X]$ в сумму неприводимых представлений группы $G$. Это сразу позволяет сказать, что в случае простой групшы $G$ почти все автоморфизмы, удовлетворяюшие условию (iii), эквивалентны инволюции Вейля. Исключения будут перечислены в теореме 3 .

Для сферических пар с непростой группой $G$ придется разбирать каждую пару отдельно, поэтому приведем здесь их классификацию.

ОпредЕлЕниЕ 7. Сферическая пара $(G, H)$ называется главной, если $Z(H)^{0}=$ $Z=\left(G^{1} \cap Z\right) \times \cdots \times\left(G^{m} \cap Z\right)$, где $G^{i}$ - простые множители группы $G$.

По поводу строения неглавных пар см. [10].

В таблице 1 перечислены все неразложимые главные несимметрические сферические пары с полупростой, но не простой, группой $G$ с точностью до локального изоморфизма.

ТАБЛИЦА 1

\begin{tabular}{|l|l|l|l|}
\hline & $G$ & $H$ & вложение $H$ в $G$ \\
\hline 1 & $\mathrm{Sp}_{2 n+4} \times \mathrm{Sp}_{4}$ & $\mathrm{Sp}_{2 n} \times \mathrm{Sp}_{4}$ & $(u, v) \rightarrow(u \oplus v, v)$ \\
\hline 2 & $\mathrm{SL}_{n} \times \mathrm{SL}_{n+1}$ & $\mathrm{SL}_{n} \times \mathbb{C}^{*}(n>1)$ & $(u, \lambda) \rightarrow\left(u, \lambda u \oplus \lambda^{-n}\right)$ \\
\hline 3 & $\mathrm{SO}_{n+1} \times \mathrm{SO}_{n}$ & $\mathrm{SO}_{n}(n>4)$ & $u \rightarrow(u, u)$ \\
\hline 4 & $\mathrm{SL}_{n+2} \times \mathrm{Sp}_{2 m}$ & $\mathrm{SL}_{2} \times \mathrm{SL}_{n} \times \mathrm{Sp}_{2 m-2} \times \mathbb{C}^{*}$ & $\begin{array}{c}(u, v, w, \lambda) \rightarrow \\
\left(\lambda^{n} u \oplus \lambda\right.\end{array}$ \\
\hline 5 & $\mathrm{SL}_{n+2} \times \mathrm{Sp}_{2 m}$ & $\mathrm{SL}_{2} \times \mathrm{SL}_{n} \times \mathrm{Sp}_{2 m-2}(n>2)$ & $\begin{array}{c}(u, v, w) \rightarrow \\
(u \oplus v, u \oplus w)\end{array}$ \\
\hline 6 & $\mathrm{Sp}_{2 n+2} \times \mathrm{Sp}_{2 m}$ & $\mathrm{Sp}_{2 n} \times \mathrm{Sp}_{2} \times \mathrm{Sp}_{2 m-2}$ & $\begin{array}{c}(u, v, w) \rightarrow \\
(u \oplus v, v \oplus w)\end{array}$ \\
\hline 7 & $\mathrm{Sp}_{2 n} \times \mathrm{Sp}_{4} \times \mathrm{Sp}_{2 m}$ & $\mathrm{Sp}_{2 n-2} \times \mathrm{Sp}_{2} \times \mathrm{Sp}_{2} \times \mathrm{Sp}_{2 m-2}$ & $\begin{array}{c}(u, v, w, z) \rightarrow \\
(u \oplus v, v \oplus w, w \oplus z)\end{array}$ \\
\hline 8 & $\mathrm{Sp}_{2 n} \times \mathrm{Sp}_{2 m} \times \mathrm{Sp}_{2 k}$ & $\mathrm{Sp}_{2 n-2} \times \mathrm{Sp}_{2 m-2} \times \mathrm{Sp}_{2 k-2} \times \mathrm{Sp}_{2}$ & $\begin{array}{c}(u, v, w, z) \rightarrow \\
(u \oplus z, v \oplus z, w \oplus z)\end{array}$ \\
\hline
\end{tabular}

Здесь $n, m, k$ - натуральные числа; если индекс в обозначении группы равен нулю, то она считается единичной.

Теорема 3. В таблице 2 перечислены с точностью до локального изоморфизма все неразложимые главные сферические пары, у которых не все правильные автоморфизмы әквивалентны инволюиии Вейля. Для кажсдй парь указаны все ее правильные автоморфизмы с точностью до әквивалентности.

ТАБЛИЦА 2

\begin{tabular}{|c|c|c|}
\hline & $(G, H)$ & $\sigma \in \operatorname{Aut}(G, H)$ \\
\hline 1 & $\begin{array}{l}\left(\mathrm{SL}_{p+q}, \mathrm{~S}\left(\mathrm{GL}_{p} \times \mathrm{GL}_{q}\right)\right),\left(E_{6}, \mathrm{Spin}_{10} \cdot \mathbb{C}^{*}\right) \\
\left(E_{6}, \mathrm{SL}_{6} \times \mathrm{SL}_{2}\right),\left(\mathrm{SO}_{4 n+2}, G L_{2 n+1}\right)\end{array}$ & id, $\theta$ \\
\hline 2 & $\left(\mathrm{SO}_{2(p+q)}, \mathrm{SO}_{2 p} \times \mathrm{SO}_{2 q}\right)(p>q)$ & id, $a\left(I_{1}\right)$ \\
\hline 3 & $\left(\mathrm{SO}_{2(p+q)}, \mathrm{SO}_{2 p+1} \times \mathrm{SO}_{2 q-1}\right)(p \geqslant q)$ & $\mathrm{id}, \varphi$ \\
\hline 4 & $(H \times H, H)$ & $\theta, \varphi$ \\
\hline
\end{tabular}

Здесь $I_{1}=\operatorname{diag}(-1,1, \ldots 1)$. Символ точка $(\cdot)$ обозначает локально прямое произведение групп. 
ДоказАтельство. Рассмотрим сначала пары с простой группой $G$. Для не указанных в таблице 2 пар условие (iii) вьполнено только в одной компоненте связности группы Aut $G$, а именно в той, которая содержит инволюцию Вейля. Для указанных в таблице 2 пар условие (iii) выполнено в двух компонентах связности группы Aut $\mathfrak{g}$ (см. [7]). Каждый из указанных автоморфизмов лежит в нормализаторе $H$. По теореме 2 они являются правильными.

Для симметрического однородного пространства $(H \times H) / H$ ненулевые $H$-инвариантные векторы есть во всех неприводимых представлениях группы $H \times H$ вида $R\left(\pi_{i}(1) \cdot \pi_{i}(2)^{*}\right)$, здесь $\pi_{i}-$ фундаментальные веса группы $H$. Пусть автоморфизм $\Psi$ сохраняет диагональ произведения и удовлетворяет условию (iii). Возможны два случая:

1) $\Psi(p, q)=(\psi(p), \psi(q))$; тогда $R\left(\pi_{i}\right)^{\psi}=R\left(\pi_{i}\right)^{*}$ для любого $i$ и $\Psi$ эквивалентен $\theta$;

2) $\Psi(p, q)=(\psi(q), \psi(p))$; тогда $R\left(\pi_{i}\right)^{\psi}=R\left(\pi_{i}\right)$ для любого $i$ и $\Psi$ эквивалентен $\varphi$.

Для указанных в таблице 1 пар все правильные автоморфизмы эквивалентны инволюции Вейля. В самом деле, как уже известно, нельзя переставлять простые сомножители $G^{i}$ группы $G$. Обозначим проекцию группы $H$ на $G^{i}$ через $H^{i}$. Любой правильный автоморфизм имеет вид $\sigma=\sigma^{1} \times \sigma^{2} \times \sigma^{3}$ (или $\sigma^{1} \times \sigma^{2}$ ), где $\sigma^{i}-$ правильный автоморфизм пары $\left(G^{i}, H^{i}\right)$.

В случаях $1,6,7$ и 8 у каждой из простых компонент нет внешних автоморфизмов.

В случае 5 на первой компоненте можно взять только инволюцию Вейля, а у второй компоненты нет внешних автоморфизмов.

В случаях 2 и 3 нетрудно найти неприводимые представления группы $G$, в каждом из которых содержится ненулевой $H$-инвариантный вектор, позволяюшие убедиться в том, что условие (iii) выполнено только в одной компоненте связности группы Aut $\mathfrak{g}$.

\begin{tabular}{|l|l|}
\hline Случай & Представления \\
\hline 2) & $\pi_{1}^{n}(1) \cdot \pi_{n}^{n+1}(2)$ \\
\hline 3) $n=7$ & $\pi_{4}(1) \cdot \pi_{3}(2), \pi_{3}(1) \cdot \pi_{3}(2)$ \\
$n=8$ & $\pi_{4}(1) \cdot \pi_{3}(2), \pi_{4}(1) \cdot \pi_{4}(2)$ \\
$n \neq 7,8$ & $\pi_{k}(1) \cdot \pi_{p}(2), k=[(n+1) / 2], p=[n / 2]$ \\
\hline
\end{tabular}

В случае 4 тождественньй автоморфизм не является правильньм, так как существует такой однородньй многочлен $f \in \mathbb{C}[\mathfrak{g} / \mathfrak{h}]^{H}$, что $\operatorname{deg} f$ - нечетное число. Действительно, рассмотрим $H$-инвариантное подпространство

$$
V \subset \mathfrak{g} / \mathfrak{h}, \quad V=\mathfrak{s l}_{2} \oplus L_{2, n} \oplus L_{n, 2} \subset \mathfrak{s l}_{n+2}
$$

$\left(L_{p, q}-\right.$ пространство комплексных матриц размера $\left.p \times q\right)$. Пусть $\xi=(X, A, B) \in V$ $\left(X \in \mathfrak{s l}_{2}, A \in L_{2, n}, B \in L_{n, 2}\right), h=(u, v, w, \lambda) \in H$ (порядок элементов $u, v$, $w, \lambda$ такой же, как в таблице 1$)$. Тогда $h \xi=\left(u X u^{-1}, \lambda^{n+2} u A v^{-1}, \lambda^{-n-2} v B u^{-1}\right)$. Пусть вектор $\eta$ лежит в $H$-инвариантном дополнении к $V$. Положим $f(\xi+\eta)=$ $f(\xi)=\operatorname{tr}(X A B)$. Тогда $f \in \mathbb{C}[\mathfrak{g} / \mathfrak{h}]^{H}$, причем $\operatorname{deg} f=3$.

Теперь опишем с точностью до эквивалентности правильные автоморфизмы неглавных пар. 
Пусть $(G, H)$ - неглавная неразложимая сферическая пара. Тогда $\mathfrak{g}=\bigoplus \mathfrak{g}_{i}$, $\mathfrak{h}=\bigoplus \mathfrak{h}_{i}^{\prime} \oplus \mathfrak{z}$, где $\left(\mathfrak{g}_{i}, \mathfrak{h}_{i}\right)-$ неразложимая главная сфрерическая пара, причем $\operatorname{dim} \mathfrak{z}_{i}=1$ для любого $i$ и $\mathfrak{z} \subset \bigoplus \mathfrak{z}_{i}$. (Здесь $\mathfrak{h}_{j}^{\prime}-$ коммутант алгебры $\mathfrak{h}_{i}$, а $\mathfrak{z} i$ - ее центр.)

Уже доказано, что правильные автоморфизмы сохраняют нормальные подгруппы. Для любого $i$ должен найтись элемент $\xi \in \mathfrak{h}^{\perp} \cap \mathfrak{z}(\mathfrak{h})=\mathfrak{z}^{\perp} \cap \mathfrak{z}(\mathfrak{h})$ с ненулевой проекцией на подалгебру $\mathfrak{g}_{i}$ (иначе пара окажется разложимой). Орбита $H \xi=\{\xi\}$ замкнута. Следовательно, чтобы задать правильный автоморфизм алгебры $\mathfrak{g}$, необходимо и достаточно в каждой связной компоненте группы Aut g указать правильный автоморфизм $\sigma_{i}$, умножающий вектор из з $i$ на -1 , если, конечно, такой автоморфизм там есть.

ПРЕДЛОЖЕНИЕ 4. С точностью до әквивалентности все правильные автоморфизмы неглавной неразложимой сферической пары $(G, H)$ имеют следующий вид: $\sigma=\sigma_{1} \times \cdots \times \sigma_{n}$, әде

$$
\sigma_{i}=\left\{\begin{array}{l}
\theta_{i}, \quad \text { ecли }\left(\mathfrak{g}_{i}, \mathfrak{h}_{i}\right) \neq\left(\mathfrak{s o}_{2 n+2}, \mathfrak{s o}_{2} \oplus \mathfrak{s o}_{2 n}\right),\left(\mathfrak{s l}_{2 n}, \mathfrak{s}\left(\mathfrak{g l}_{n} \oplus \mathfrak{g l}_{n}\right)\right) \\
a\left(I_{1}\right) \text { или } a\left(I_{1,2 n+2}\right), \quad \text { ecлu }\left(\mathfrak{g}_{i}, \mathfrak{h}_{i}\right)=\left(\mathfrak{s o}_{2 n+2}, \mathfrak{s o}_{2} \oplus \mathfrak{s o}_{2 n}\right) ; \\
a\left(S_{n}\right) \text { или } \theta_{i}, \quad \text { еслu }\left(\mathfrak{g}_{i}, \mathfrak{h}_{i}\right)=\left(\mathfrak{s l}_{2 n}, \mathfrak{s}\left(\mathfrak{g l}_{n} \oplus \mathfrak{g l}_{n}\right)\right) .
\end{array}\right.
$$

Здесь $I_{1}=\operatorname{diag}(-1,1, \ldots, 1), I_{1,2 n+2}=\operatorname{diag}(-1,1, \ldots, 1,-1), S_{n}=\left(\begin{array}{cc}0 & E_{n} \\ E_{n} & 0\end{array}\right)$.

ДокАЗАТЕЛьство. Если пара $\left(\mathfrak{g}_{i}, \mathfrak{h}_{i}\right)$ не совпадает ни с одной из пар:

$$
\left(\mathfrak{s l}_{p+q}, \mathfrak{s}\left(\mathfrak{g l}_{p} \oplus \mathfrak{g l}_{q}\right)\right),\left(E_{6}, \mathfrak{s o}_{10} \oplus \mathbb{C}\right),\left(\mathfrak{s o}_{2 n+2}, \mathfrak{s o}_{2 n} \oplus \mathfrak{s o}_{2}\right)
$$

то, как следует из теоремы $3, \sigma_{i}=\theta_{i}$. У каждой из перечисленных пар есть два неэквивалентных правильных автоморфизма. При этом у пар $\left(E_{6}, \mathfrak{s o}_{10} \oplus \mathbb{C}\right)$ и $\left(\mathfrak{s l}_{p+q}, \mathfrak{s}\left(\mathfrak{g l}_{p} \oplus \mathfrak{g l}_{q}\right)\right)$ при $p \neq q$ нет правильных внутренних автоморфизмов, умножающих вектор из центра алгебры $\mathfrak{h}$ на -1 , а все внешние правильные автоморфизмы эквивалентны инволюции Вейля.

\section{§5. Вещественные слабо симметрические пространства}

В этом параграфе $G$ - вешественная полупростая группа Ли, $G_{\mathbb{C}}-$ ее комплексификация. Мы будем предполагать, что группа $G_{\mathbb{C}}$ односвязна. Результаты данного параграфа сводят классификацию правильных автоморфизмов групшы $G$ к классификации правильных автоморфизмов компактной вещественной формы групшы $G_{\mathbb{C}}$.

ОПРЕДЕЛЕНИЕ 8. Слабо симметрическое пространство $G / K$ называется неразложсимым, если оно не представимо в виде $G^{1} / K^{1} \times G^{2} / K^{2}$, где пространства $G^{1} / K^{1}$ и $G^{2} / K^{2}$ слабо симметрические, $G=G^{1} \times G^{2}, K=K^{1} \times K^{2}$.

Пусть $\left(G=G^{1} \times G^{2}, K=K^{1} \times K^{2}\right)$ - разложимая слабо симметрическая пара. Тогда ее комплексификация $\left(G_{\mathbb{C}}=G_{\mathbb{C}}^{1} \times G_{\mathbb{C}}^{2}, H=H^{1} \times H^{2}\right)$ - разложимая сферическая пара, для которой все правильные автоморфизмы сохраняют подгруппы $G_{\mathbb{C}}^{1}$ и $G_{\mathbb{C}}^{2}$. Следовательно, всякий правильный автоморфизм $\sigma$ групшы $G$ является произведением автоморфизмов $\sigma_{1}$ и $\sigma_{2}$ групп $G^{1}$ и $G^{2}$ соответственно. Как и в комплексном случае, верно следующее 
ПРЕДЛОЖЕНИЕ 5. Автоморфизм $\sigma=\sigma^{1} \times \sigma^{2}$ правильный тогда и только тогда, когда $\sigma^{1}$ и $\sigma^{2}$ правильные.

Следовательно, достаточно изучить правильные диффеоморфизмы неразложимых слабо симметрических пространств. Нам понадобятся некоторые факты, касающиеся классификации этих пространств (см. [10]).

1) Любое неразложимое слабо симметрическое пространство является вешественной формой неразложимого сферического пространства.

2) Все некомпактњые неразложимые слабо симметрические пространства получаются следуюшим образом: $G=\left(G_{\mathbb{C}}^{\tau \varphi}\right)^{0}, K=H^{\tau}$, где $H$ - редуктивная сферическая подгруппа группы $G, \varphi$ - инволюция групшы $G_{\mathbb{C}}$, ограничение которой на $H$ - тождественный автоморфизм, а $\tau$ - компактная вещественная структура, коммутируюшая с $\varphi$ и сохраняюшая $H$.

Лемма 5. Пусть $\sigma \in$ Aut $G_{\mathbb{C}}-$ правильный автоморфизм комплексной неразложсмой сферической пары $\left(G_{\mathbb{C}}, H\right)$. Тогда в описанных выше обозначения $\sigma \varphi=\varphi \sigma$.

ДокАЗАтЕльство. Достаточно доказать, что $\sigma\left(G^{\varphi}\right)=G^{\varphi}$. Заметим, что обе эти подгрупшы содержат группу $H$. Для симметрических пар утверждение тавтологическое. Для всех пар с простой группой $G_{\mathbb{C}}$, кроме пары $\left(\operatorname{Spin}_{8}, G_{2}\right)$, сушествует единственная подгруппа вида $G_{\mathbb{C}}^{\varphi}$, содержащая $H$ (см. [10]).

Для пары $\left(\operatorname{Spin}_{8}, G_{2}\right)$ автоморфизм $\sigma$ внутренний. Следовательно, инволюции $\varphi$ и $\sigma \varphi \sigma^{-1}$ лежат в одной компоненте связности группы $\mathrm{Aut}\left(\mathrm{Spin}_{8}\right)$. Это означает, что эти инволюции оставляют на месте один и тот же центральный элемент группы $\operatorname{Spin}_{8}$ (отличный от единичного). Предположим, что $\varphi \neq \sigma \varphi \sigma^{-1}$. Тогда и у группы $\mathrm{SO}_{8}=\mathrm{Spin}_{8} / \mathbb{Z}_{2}$ тоже есть две инволюции, действующие тривиально на сфиерической подгруппе $G_{2}$. А это не так (см. [10]).

Пусть $G_{\mathbb{C}}$ полупроста и $G_{\mathbb{C}}=G_{\mathbb{C}}^{1} \times \cdots \times G_{\mathbb{C}}^{n}$, где $G_{\mathbb{C}}^{i}-$ простые группы. Как мы уже знаем, автоморфизм $\sigma$ не переставляет компоненты несимметрических пар. Если $\sigma$ и $\varphi$ сохраняют $G_{\mathbb{C}}^{i}$, то на ней они коммутируют. Если же инволюция $\varphi$ переставляет две компоненты $G_{\mathbb{C}}^{i}$ и $G_{\mathbb{C}}^{j}$ (это возможно только для пары $\left.\left(\mathrm{Sp}_{2} \times \mathrm{Sp}_{2} \times \mathrm{Sp}_{2 k}, \mathrm{Sp}_{2} \times \mathrm{Sp}_{2 k-2}\right)\right)$, то диагональ произведения $G_{\mathbb{C}}^{i} \times G_{\mathbb{C}}^{j}$ содержится в проекции $H$ на $G_{\mathbb{C}}^{i} \times G_{\mathbb{C}}^{j}$, т.е. автоморфизм $\sigma$ сохраняет множество неподвижных точек инволюции.

СЛЕДСТВИЕ. Пусть $\left(G_{1}, K\right)$ u $\left(G_{2}, K\right)$ - слабо симметрические парь, соответствующие сферической паре $\left(G_{\mathbb{C}}, H\right)$. Тогда множсества правильных автоморфизмов групп $G_{1}$ и $G_{2}$, рассматриваемые как подмножества групnвъ $\operatorname{Aut}\left(G_{\mathbb{C}}, H\right)$, coвпадают.

ДокАЗАТЕльство. По теореме 1 существует биекция между множеством правильных автоморфизмов групшы $G_{i}(i=1,2)$ и множеством правильных автоморфизмов пары $\left(G_{\mathbb{C}}, H\right)$, сохраняющих вешественную форму $G_{i}$. Можно считать, что группа $G_{1}$ компактна. Тогда если $\sigma-$ правильньй автоморфизм пары $\left(G_{\mathbb{C}}, H\right)$, то

$$
\sigma\left(G_{2}\right)=G_{2} \Longleftrightarrow \sigma \tau \varphi=\tau \varphi \sigma \Longleftrightarrow \sigma \tau=\tau \sigma \Longleftrightarrow \sigma\left(G_{1}\right)=G_{1} .
$$

\section{§6. Классификация правильных автоморфизмов в вешественном случае и ее следствие}

Пусть $\sigma$ - правильный автоморфизм пары $(G, K)$. Автоморфизм $a(g) \sigma\left(g \in G_{\mathbb{C}}\right)$ является правильным автоморфизмом пары $(G, K)$ тогда и только тогда, когда 
$g \in N(K) \cap N(G)$. Если $N(G) \neq G$, то эквивалентные правильные автоморфизмы пары $\left(G_{\mathbb{C}}, H\right)$, сохраняющие $G$, могут быть не эквивалентны как правильные автоморфизмы пары $(G, K)$. Но для компактной вещественной формы $N(G)=G$; следовательно, правильные автоморфизмы пары $(G, K)$ эквивалентны как автоморфизмы группы $G_{\mathbb{C}}$ тогда и только тогда, когда они эквивалентны как автоморфизмы групшы $G$.

Предположим теперь, что группа $G$ компактна. Для любого полупростого автоморфизма $\psi$ групшы $G_{\mathbb{C}}$ найдется компактная вешественная структура $\tau$, коммутируюшая с $\psi$. Любой правильный автоморфизм групшы $G_{\mathbb{C}}$ эквивалентен либо инволюции, либо тождественному автоморфизму. Поэтому можно считать, что указанные в $\S 4$ автоморфизмы являются правильными автоморфизмами соответствующих компактных групп. Все правильные автоморфизмы получаются умножением этих автоморфизмов на элементы группы $\operatorname{Int}(G, K)$.

В частности, любой правильный автоморфизм группы $G$ имеет вид: $\sigma=a(n) \delta$, где $n \in N(K)$, а $\delta$ - инволютивный правильный автоморфизм. Но тогда

$$
\sigma^{2}=a(n \delta(n))=a\left(n\left(n^{-1} k\right)\right)=a(k), \quad \text { где } k \in K,
$$

т.е. диффеоморфизм $s^{2}$, соответствующий автоморфизму $\sigma^{2}$, действует на многообразии $M$ как $k$. Следовательно, если рассматривать группу $\widehat{G}$ как подгрупу группы $\operatorname{Diff}(M)$, то условие $s^{2} \in G$ будет следствием того, что диффеоморфизм $s$ правильный. Таким образом, в случае полупростой группы $G$ данное нами вначале определение слабо симметрического пространства (см. §1) оказывается эквивалентньм оригинальному определению Сельберга.

Автор благодарен своему научному руководителю Э.Б. Винбергу за постановку задачи и внимательное отношение к работе на всех ее стадиях, а также Д.А. Тимашёву за ряд полезных замечаний.

\section{Список литературы}

1. Selberg A. Harmonic analysis and discontinuous groups in weakly symmetric Riemannian spaces with applications to Dirichlet series // J. Indian Math. Soc. 1956. V. 20. P. 47-87.

2. Akhiezer D. N., Vinberg E. B. Weakly symmetric spaces and spherical varieties // Transformation Groups. 1999. V. 4. P. 12-46.

3. Berndt J., Prüfer F., Vanhecke L. Symmetric-like Riemannian manifolds and geodesic symmetries // Proc. Roy. Soc. Edinburgh Sect. A. 1995. V. 125. P. 265-282.

4. Винберг Э.Б., Онищик А. Л. Семинар по группам Ли и алгебраическим группам. М.: Наука, 1988.

5. Винберг Э. Б. Коммутативные однородные пространства и коизотропные действия // УМH. 2001. T. 56. № 1. C. 3-62.

6. Panyushev D. Complexity and rank of homogeneous spaces // Geom. Dedicata. 1990. V. 34. P. 249-269.

7. Krämer $M$. Sphärische Untergruppen in kompakten zusammenhängenden Liegruppen // Compositio Math. 1979. V. 38. P. 129-153.

8. Brion M. Classification des espaces homogènes sphériques // Compositio Math. 1987. V. 63. P. $189-208$.

9. Микитюк И.В. Об интегрируемости инвариантных гамилтоновых систем с однородными конфигурационными пространствами // Матем. сб. 1986. Т. 129. С. 514-534.

10. Якимова О.С. Слабо симметрические пространства полупростых групп Ли // Вестн. МГУ. Сер. 1. Матем., мех. 2002. № 2 (в печати). 